\title{
Subaru Coronagraphic Extreme-AO (SCExAO) wavefront control: current status and ongoing developments
}

Ananya Sahoo, Olivier Guyon, Christophe S. Clergeon, Nour Skaf, Yosuke Minowa, et al.

Ananya Sahoo, Olivier Guyon, Christophe S. Clergeon, Nour Skaf, Yosuke Minowa, Julien Lozi, Nemanja Jovanovic, Frantz Martinache, "Subaru Coronagraphic Extreme-AO (SCExAO) wavefront control: current status and ongoing developments," Proc. SPIE 10703, Adaptive Optics Systems VI, 1070350 (11 July 2018); doi: 10.1117/12.2314333

Event: SPIE Astronomical Telescopes + Instrumentation, 2018, Austin, Texas, United States 


\title{
Subaru Coronagraphic Extreme-AO (SCExAO) Wavefront Control: Current Status and Ongoing Developments
}

\author{
Ananya Sahoo ${ }^{\mathrm{a}, \mathrm{b}}$, Olivier Guyon ${ }^{\mathrm{a}, \mathrm{e}, \mathrm{f}, \mathrm{g}}$, Christophe S. Clergeon ${ }^{\mathrm{a}}$, Nour Skaf ${ }^{\mathrm{a}}$, Yosuke Minowa ${ }^{\mathrm{a}}$, Julien \\ Lozi $^{\mathrm{a}}$, Nemanja Jovanovic ${ }^{\mathrm{c}}$, Frantz Martinanche ${ }^{\mathrm{d}}$ \\ ${ }^{a}$ Subaru Telescope, National Astronomical Observatory of Japan, 650 North A'ohoku Place, Hilo, HI 96720, U.S.A.

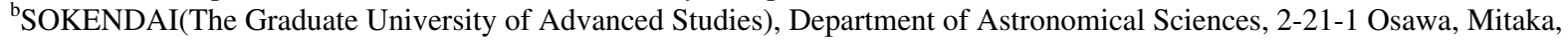 \\ Tokyo 181-8588, Japan \\ ${ }^{\mathrm{c} C}$ Caltech Optical Observatories, California Institute of Technology, 1200 E. California Boulevard,Pasadena, CA 91125, U.S.A \\ ${ }^{\mathrm{d}}$ Observatoire de la Cote dAzur, 96 Boulevard de lObservatoire, 06300 Nice, France \\ ${ }^{\mathrm{e}}$ College of Optical Sciences, University of Arizona, Tucson, AZ 85721, U.S.A. \\ ${ }_{\mathrm{f}}^{\mathrm{f}} \mathrm{Jet}$ Propulsion Laboratory, 4800 Oak Grove Drive, MS 183-901, Pasadena, CA 91109, U.S.A. \\ ${ }^{\mathrm{g}}$ Astrobiology Center of NINS, 2-21-1, Osawa, Mitaka, Tokyo, 181-8588, Japan
}

\begin{abstract}
Exoplanet imaging requires excellent wavefront correction and calibration. At the Subaru telescope this is achieved using the 188-element facility adaptive optics system(AO188) feeding the Subaru Coronagraphic Extreme Adaptive Optics (SCExAO) instrument; a multipurpose instrument built to deliver high contrast images of planets and disks around nearby stars. AO188 offers coarse correction while SCExAO performs fine correction and calibration of 1000 modes. The full system achieves $\sim 90 \%$ Strehl Ratio in H-band and diffraction limited images. A new Real Time Computer allowing higher performance between SCExAO and AO188 is currently implemented. Future upgrades will include a new Pyramid Wavefront Sensor and (64x64) DM to achieve extreme AO correction inside AO188. We are progressing in the development of predictive control and sensor fusion algorithms across the system to improve performance and calibration. With the new upgrades, SCExAO will be able to image giant plan-ets in reflected light with Subaru and validate technologies necessary to image habitable Earth-like planets with the Thirty Meter Telescope (TMT).
\end{abstract}

Keywords: adaptive optics, exoplanet, wavefront sensors, astronomical instrumentations, wavefront control.

\section{Introduction}

Direct imaging of nearby exoplanets and the circumstellar disks can give crucial information about their atmospheric composition and can refine our understanding of planetary formation. These planetary mass companions typically within small angular separations are between 4 and 10 orders of magnitude fainter than their host star and makes direct imaging to be challenging. Extreme AO with coronagraphs are deployed in ground based telescopes to mitigate these challenges. The Subaru Coronagraphic Extreme Adaptive (SCExAO) is one such instruments. ${ }^{1}$ It combines multiple wavefront sensor techniques with several stateof-the-art coronagraphs such as vector vortex coronagraph, ${ }^{2}$ Phase Induced Amplitude Apodization (PIAA) ${ }^{3}$ coronagraph to push the inner working angle (IWA) to less than $\lambda / D$. SCExAO takes light from the 188element facility adaptive optics system $(\mathrm{AO} 188)^{4}$ which gives a partial correction of low order modes and feeds an integral field spectrograph CHARIS(Coronagraphic High Angular Resolution Spectrograph) at the IR Nasmyth platform of the Subaru Telescope. AO188 is comprised of a curvature based wavefront sensor which runs at $1 \mathrm{KHz}$ with Avalanch Photodiodes (APDs) and a 188-actuator bi-morph deformable mirror (DM) to achieve Strehl ratios (SR) up to 30-40\% in H band. A wavefront control system is the heart of operation of any extreme AO systems and act as key for high contrast imaging. SCExAO comprises of a pyramid based high order wavefront sensor (PyWFS) to measure higher order modes up to 1000 and a Lyot based low order wavefront sensor to identify low order modes such as tip-tilt, coma, defocus, astigmatism etc. The wavefront correction is performed by a MEM technology based 2000-actuator Deformable Mirror (DM) running at upto $3.5 \mathrm{KHz}$. It is operated by a wavefront control algorithm using high sensitivity PyWFS to suppress the speckle noise. The current optical system is capable of achieving $\sim 90$ Strehl Ratio(SR) in H-band and delivers diffraction limited images in visible light. Future plans include upgrading AO188's software and hardware components. We are currently implementing a new Real Time Computer (RTC) to

\footnotetext{
Adaptive Optics Systems VI, edited by Laird M. Close, Laura Schreiber,

Dirk Schmidt, Proc. of SPIE Vol. 10703, $1070350 \cdot$ @ 2018 SPIE

CCC code: $0277-786 \mathrm{X} / 18 / \$ 18 \cdot$ doi: $10.1117 / 12.2314333$
} 
allow for higher performance real time communications between SCExAO and AO188. Future upgrades will include new PyWFS and new (64x64)DM to achieve extreme AO correction inside AO188, so that SCExAO's DM can be focused on the fine control and calibration of speckle noise. Presently, we are progressing in the development of predictive control and sensor fusion algorithms across the system to improve performance and calibration. SCExAO acts as an ideal platform to demonstrate technologies needed for future large telescopes.

\section{SCExAO's Wavefront Control Architecture}

Wavefront control primarily comes in two stages, a wavefront sensor and then wavefront corrector. The former measures the phase error and sends commands to the later rectify these errors. Figure 1 shows a schematic representation of the overall wavefront control architecture of SCExAO. It has several loops correcting different regions of the wavefront residuals. SCExAO feeds partially corrected light (600-2500 nm) from AO188. The $2 \mathrm{k}$ deformable mirror splits the light in two parts. The visible light $(600-950 \mathrm{~nm})$ is reflected to the visible bench which hosts the PyWFS and the rest of the light The IR light that is transmitted by the dichroic to the Infrared IR bench propagates through the coronagraphs and then to the science instruments. Some of the light which is reflected from the coronagraphs is used to drive Lyot based low order wavefront sensor (LLOWFS). It corrects chromatic and non common path errors such as tip/tilt between the visible and Infrared (IR) bench.

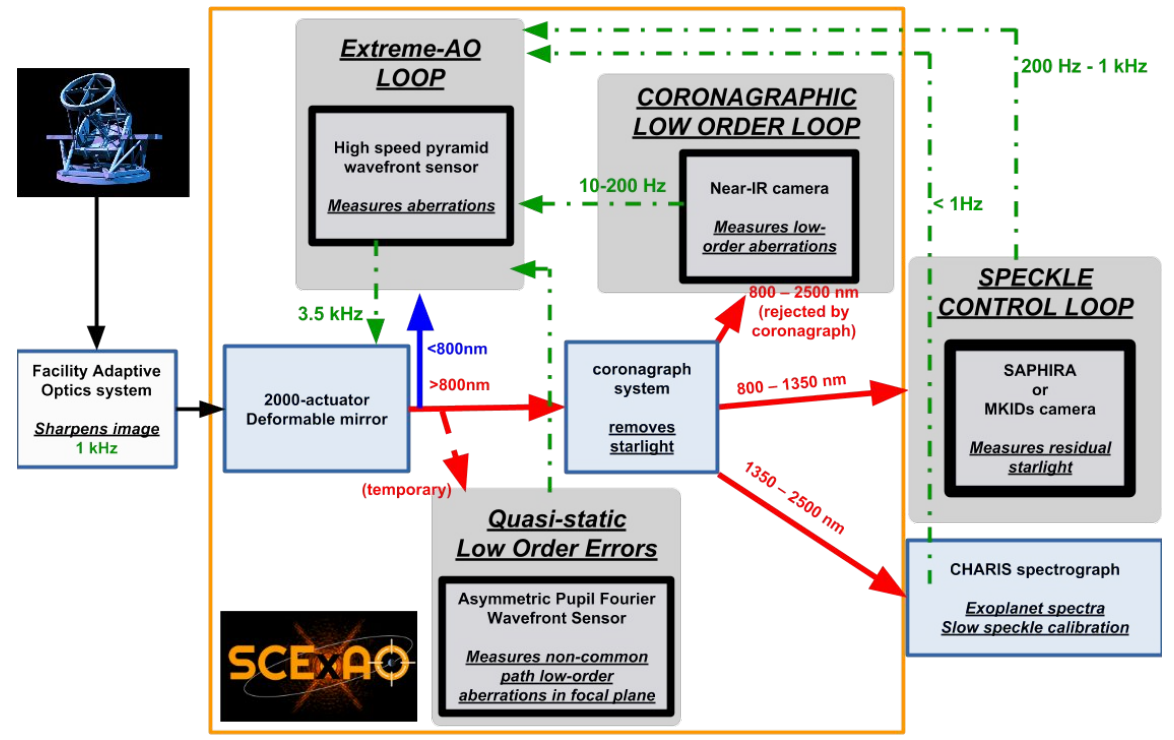

Fig 1 Schematic representation of SCExAO's wavefront control architecture

The 2k-actuator MEMs technology based deformable mirror has a controllable silicon reflective surface to correct distorted input wavefront. It is enclosed in a sealed chamber to control its environment. There are $45 \times 45: 5$ actuators across the $18 \mathrm{~mm}$ beam therefore spatial frequencies out to $22.5 \lambda / D$ from the PSF can be addressed. Figure 2 shows the actual picture of the DM installed on the SCExAO bench.

Since PyWFS has high sensitivity and large dynamic range, it chosen as the ExAO primary loop of the SCExAO's wavefront control system which sends the final commands to the DM. ${ }^{5,6}$ Other Wavefront sensors' commands to the DM , are sent as an offset to this PyWFS loop. The DM commands coming from the various sources are then co-added into a single DM map that is sent to the DM. Figure 6 shows various loop which sends command to the DM. The light in front of the PyWFS is modulated in circular trajectory using a tip tilt mirror to increase the to increase the range in which PyWFS responds linearly to 


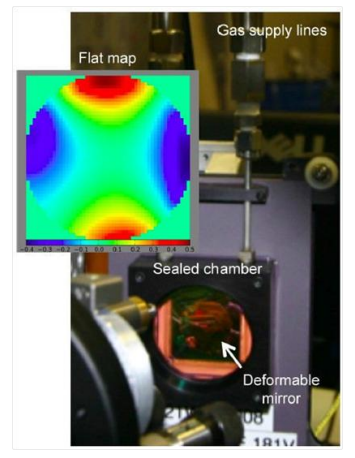

Fig 2 Deformable mirror mounted on SCExAO bench

the aberrations. Figure 3 Shows a picture of the pyramid optics. This loop corrects aberrations up to 1200 modes.
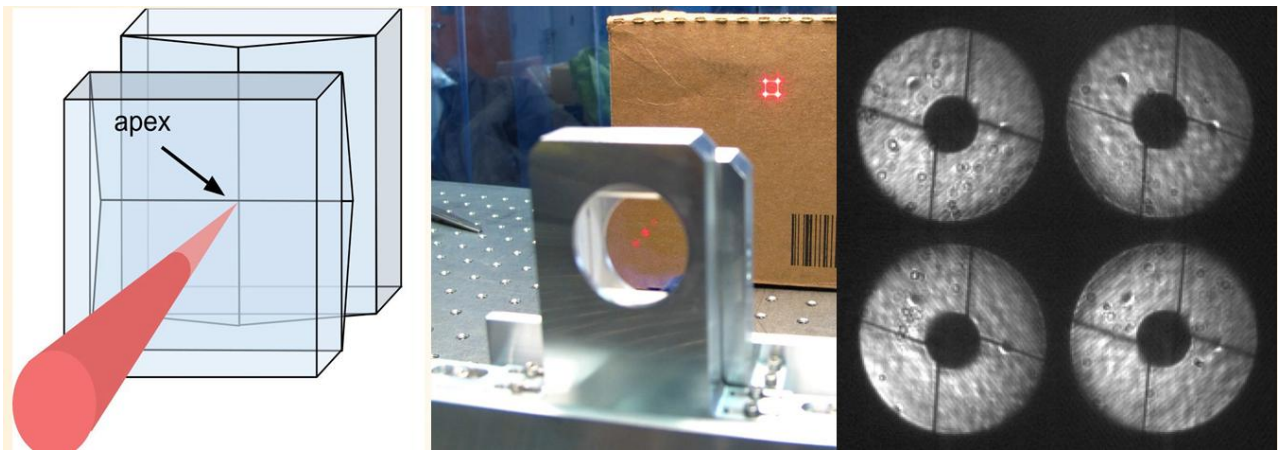

Fig 3 Image of the PyWFS prism with the four pupil images taken with camera OCAM2k

In addition to the ExAO loop, LLOWFS loop addresses the non-common path and chromatic low-order errors between the visible PyWFS and the IR coronagraphs. The light diffracted (see figure 4) by the focal plane masks of the coronagraphs is used by the LLOWFS to correct tip/tilt aberrations by taking into considerations asymmetries in the image. ${ }^{7}$

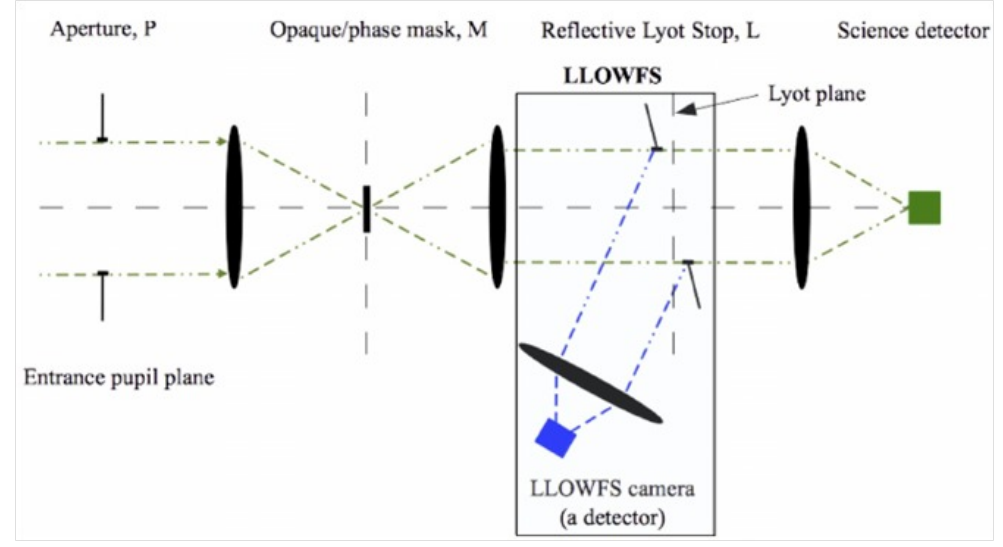

Fig 4 Schematic representation of the Lyot-based low order wavefront sensor (LLOWFS) with a high performance coronagraph and reflective Lyot stop (RLS. 
In addition to the PyWFS and LLOWFS several other wavefront sensing techniques are implemented for statics and dynamic high-order aberrations. One such technique is known as focal plane wavefront sensing. ${ }^{8}$ It is used to improve the contrast in the image by eliminating the speckles in the PSF (speckle nulling). The DM is modulated to generate speckles which interfere destructively with the speckles already present in the image. The brightest speckles are identified in the image, for which amplitudes and positions are measured. With these informations, speckles are created using sine waves on the DM, at the exact same spatial frequencies, and with similar amplitudes. The phase of the sinusoidal wave is varied. For a particular phase of the applied sinusoidal wave, the total intensity of the speckle becomes negligible. A few tens of speckles are corrected at the same time, and the control is performed in an iterative loop until the maximum contrast is reached. This method only allow the correction on one half of the speckle field when only one DM is used. Figure 5 shows the on sky result achieved with speckle nulling technique.
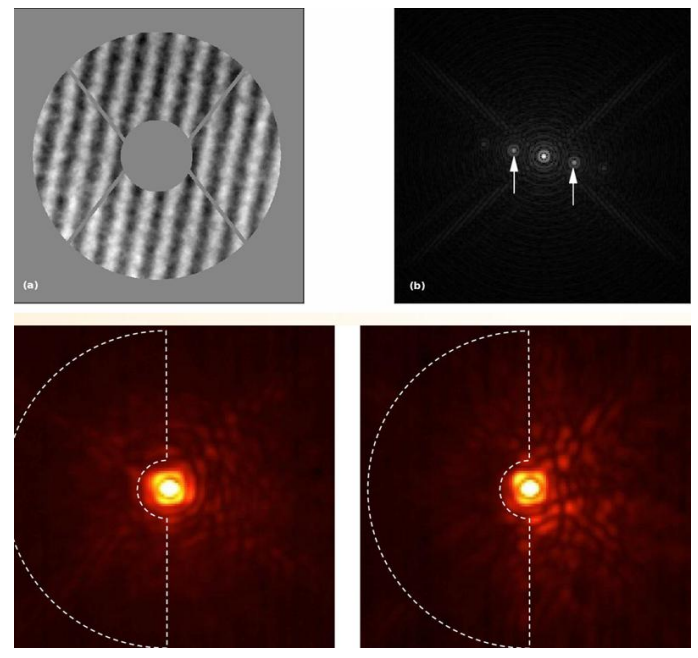

Fig 5 Top:Sinusoidal phase modulation is applied to DM to create speckles. Bottom: Left picture shows the PSF without speckle nulling and right image depicts speckle nulling performed on the region enclosed by white dashed line.

Further, the addition of artificial speckles to the focal plane image can be utilized for precision astrometry when the on-axis starlight has been suppressed post-coronagraph. Speckles can be made incoherent with the speckle in the halo by modulating their phase for superior astrometric performance. We can also measure the contrast of the companion by calibrating the flux ratio between the PSF core and the speckles. ${ }^{9}$

\section{Future Upgrade}

In order to improve the overall Subaru's AO performance, a few upgrades have been planed for AO188 and SCExAO. Presently there is a lack of communication between the two instruments. This limits us from sending any commands to the DM and transferring any telemetry between the instruments. The hardware and software of AO188 are relatively old and may obstruct us to achieve an optimal wavefront correction. A new Real Time Computer (RTC) is being currently in a phase of tests to make it compatible with the old RTC's hardware. The software upgrade for AO188 includes the use of GPUs for fast real-time computations. The curvature wavefront sensor of AO188 using APDs will be upgraded with a PyWFS, similar to the one inside SCExAO, with a First Light Imaging OCAM2K camera as the detector. The 188-actuator bimoprh DM will be replaced with a 64x64-actuator DM, twill give AO188 ExAO capabilities even without SCExAO. In this configuration, SCExAO's 2000-actuator DM would be exclusively used for speckle control to achieve higher contrast in one half of the image. A new predictive control algorithm is being implemented to reproduce future WFS measurements by linear combination of past WFS measurements for 


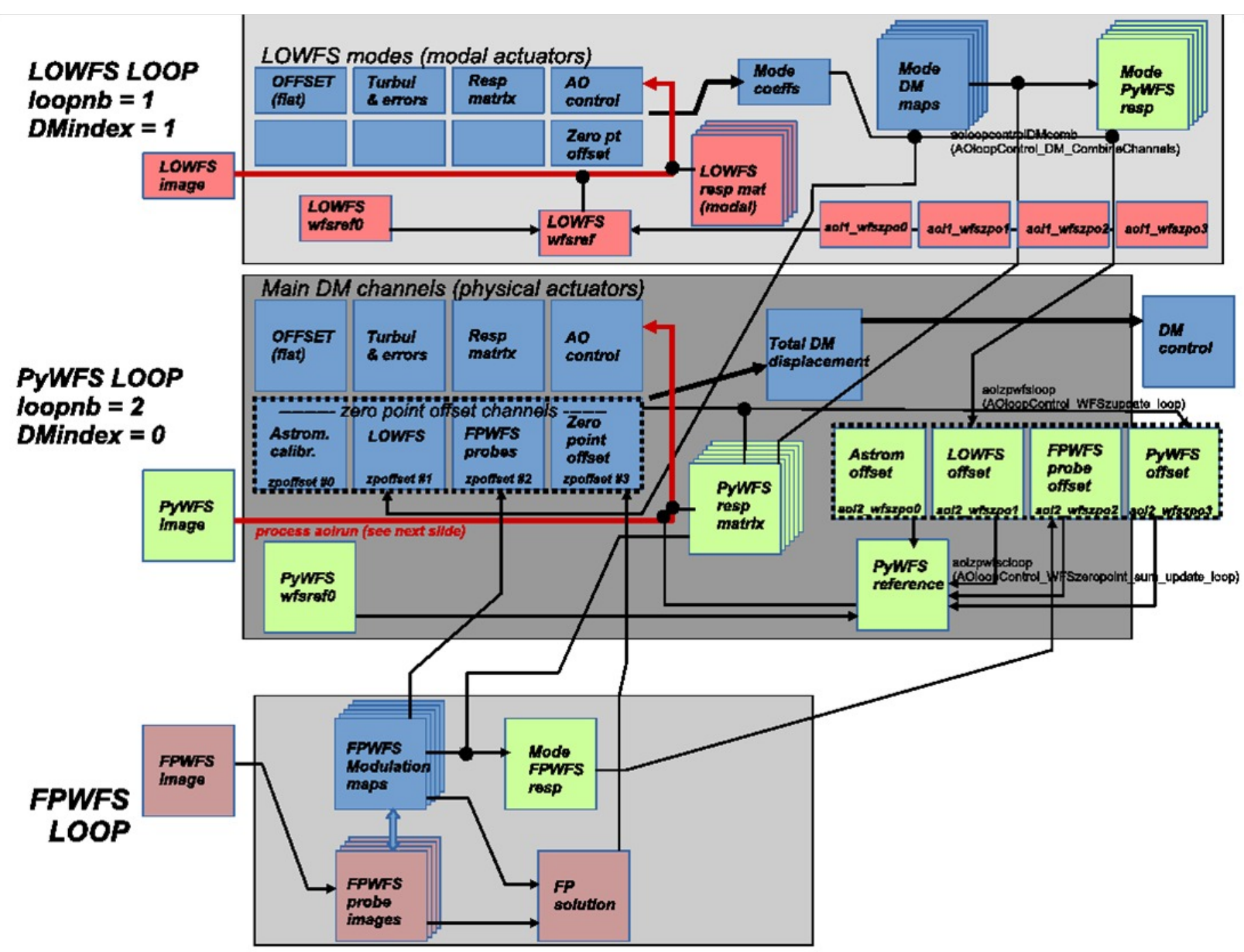

Fig 6 Schematic representation of the SCExAO's wavefront control architecture software. It shows the interactions between the various wavefront control loops. Blue boxes depicts commands in DM space, while green boxes are images in WFS space. The main loop uses the the PyWFS and sends commands to the DM. The DM commands coming from the various sources are then co-added into a single DM map that is sent to the DM.

optimal wavefront corrections. ${ }^{10}$ The hardware upgrades of SCExAO includes installation of an Infrared camera; First Light Imaging CRED-2 and a Microwave Kinetic Inductance Detector (MKID) ${ }^{11}$ which is a photon counting, energy discriminating in near Infrared. Both these cameras have a low readout noise and can be used for active speckle suppression. These upgrades will make the combination AO188 and SCExAO the ultimate testbed for a first generation high-contrast imager for future large telescopes.

\section{Conclusion}

The extreme adaptive optics system of SCExAO delivers the necessary wavefront correction and can push the detection limit for ground based telescopes to smaller inner working angles. SCExAO performs fine correction and calibration of $\sim 1000$ modes. With the ExAO loop, the current system is capable of achieving $\sim 90 \%$ Strehl Ratio in H-band and delivers diffraction limited images in visible light. There has been progress in the development of predictive control and sensor fusion algorithms across the system to improve performance and calibration. With the new upgrades SCExAO will be able to image giant planets in reflected light with Subaru and validate technologies necessary to image habitable Earth-like planets with the Thirty Meter Telescope(TMT). 


\section{References}

1 N. Jovanovic, F. Martinache, O. Guyon, et al., "The subaru coronagraphic extreme adaptive optics system: Enabling high-contrast imaging on solar-system scales," Publications of the Astronomical Society of the Pacific 127(955), 890 (2015).

2 D. Mawet, E. Serabyn, K. Liewer, et al., "The vector vortex coronagraph: Laboratory results and first light at palomar observatory," The Astrophysical Journal 709(1), 53 (2010).

3 O. Guyon, "Phase - induced amplitude apodization of telescope pupils for extrasolar terrestrial planet imaging," Astron. Astrophys. 404, 379 (2003).

4 Y. Minowa, Y. Hayano, S. Oya, et al., "Performance of subaru adaptive optics system ao188," in Proceedings of SPIE - The International Society for Optical Engineering, 7736 (2010).

5 O. Guyon, "Limits of adaptive optics for high contrast imaging," Astrophys. J. 629, 592-614 (2005).

6 C. Clergeon, O. Guyon, F. Martinache, et al., "The subaru coronagraphic extreme ao high speed and high sensitivity wavefront sensors," in Proceedings of the Third AO4ELT Conference, S. Esposito and L. Fini, Eds., INAF - Osservatorio Astrofisico di Arcetri, (Firenze) (2013).

7 G. Singh, F. Martinache, P. Baudoz, et al., "Lyot-based low order wavefront sensor for phase-mask coronagraphs: Principle, simulations and laboratory experiments," Publications of the Astronomical Society of the Pacific 126(940), 586 (2014).

8 F. Martinache, O. Guyon, N. Jovanovic, et al., "On-sky speckle nulling demonstration at small angular separation with scexao," Publications of the Astronomical Society of the Pacific 126(940), 565 (2014).

9 N. Jovanovic, O. Guyon, F. Martinache, et al., "Artificial incoherent speckles enable precision astrometry and photometry in high-contrast imaging," The Astrophysical Journal Letters 813(2), L24 (2015).

10 O. Guyon and J. Males, "Adaptive Optics Predictive Control with Empirical Orthogonal Functions (EOFs)," ArXiv e-prints (2017).

11 B. A. Mazin, B. Bumble, S. R. Meeker, et al., "A superconducting focal plane array for ultraviolet, optical, and near-infrared astrophysics," Opt. Express 20, 1503-1511 (2012). 